Western University Scholarship@Western

Centre for Decision Sciences and Econometrics

Technical Reports

1988

\title{
Continuous Implementation of Constrained Rational Expectations Equilibria
}

David Wettstein

Follow this and additional works at: https://ir.lib.uwo.ca/economicscdse_tr Part of the Economics Commons

Citation of this paper:

Wettstein, David. "Continuous Implementation of Constrained Rational Expectations Equilibria." Centre for Decision Sciences and Econometrics Technical Reports, 20. London, ON: Department of Economics, University of Western Ontario (1988). 


\section{THE CENTRE FOR DECISION SCIENCES AND ECONOMETRICS}

Continuous Implementation of Constrained

Rational Expectations Equilibria

David Wettstein

\section{TECHNICAL REPORT NO. 20 \\ JANUARY 1988}

\section{Centre For Decision Sciences And Econometrics Social Science Centre The University of Western Ontario London, Ontario N6A 5C2}

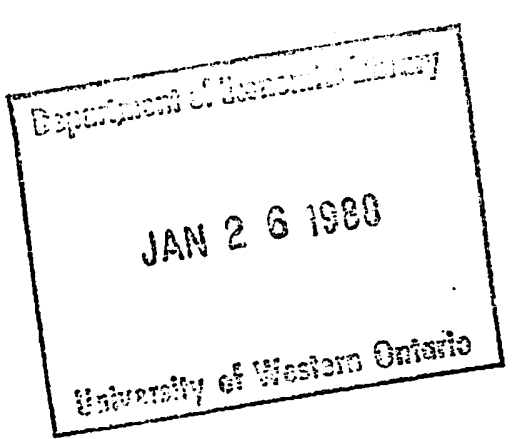


ISSN: $0831-4454$

ISBN : 0-7714-0961-3

\section{Continuous Implementation of Constrained Rational Expectations Equilibria ${ }^{*}$}

by

David Wettstein

University of Western Ontario

London, Canada

N6A 5 C2

November 1987

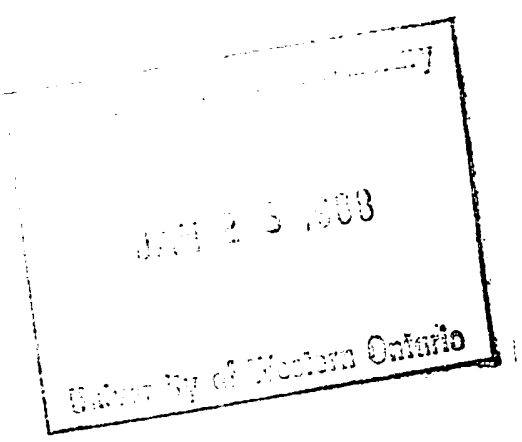

*The research reported in this paper constitutes part of my $\mathrm{Ph} . \mathrm{D}$. thesis submitted to the University of Minnesota. I wish to thank my thesis adviser Professor Leonid Hurwicz for helpful discussions. James Jordan provided comments on an earlier version. Helpful conversations with Andrew Postlewaite and David Schmeidler are gratefully acknowledged. Debbie Fretz provided able editorial assistance. 


\section{Abstract}

We investigate the implementability of the Social Choice Correspondence induced by Constrained Rational Expectations Equilibria. An "almost continuous" mechanism implementing it is constructed, provided certain conditions, one of which is Non Exclusive Information, are satisfied.

Journal of Economic Literature Classification Numbers: 025, 026 


\section{Introduction}

The theory of implementation deals with ways by which a society can achieve desired outcomes. It takes into account the strategic considerations individuals would go through when asked to provide some information or take a particular course of action and tries to overcome the resulting difficulties.

We restrict our analysis to economic environments with incomplete information. Postlewaite and scmeidler [9] and [10] provided a general framework within which one can study the implementability of various social goals. They formalized the notion of a Social Choice Correspondence (SCC) and among other results provided a set of sufficient conditions for the implementability of a given scC. Palfrey and srivastava [7] derived similar results using a slightly weaker set of assumptions. Both proofs were constructive and provided particular mechanisms carrying out the implementation. As is well known, this approach differes from related research on truthful implementation that utilizes the revelation principle; see Postlewaite and Schmeidler [10] and Palfrey and Srivastava [8] for further details.

As was the case under conditions of complete information a natural question is, what SCC's of economic interest are implementable? To see this question is indeed of interest we recall that an SCC maps environments to sets of allocations. Given a family of environments and an scc, implementing the SCC amounts to designing a mechanism whose set of equilibrium outcomes for each member of that family coincides with the set of outcomes prescribed for that member by the sCC. Thus if an scC is not implementable it implies that once strategic considerations on the part of the individuals are taken into account, as indeed they should be, a central planner would find it impossible to design a mechanism (institution) realizing it. 
One of the leading models used to characterize the competitive behavior of agents under uncertainty is Rational Expectations Equilibria (REE). REE are a close counterpart of the Walrasian equilibria in economies with complete information. A good discussion of this equilibrium notion, concentrating mainly on the existence issue, and relevant references can be found in Jordan and Radner [6].

The usual criticism levelled against the Walrasian paradigm is equally applicable here. With a finite number of agents, the strategic aspects of individualistic behavior do matter; this however is not incorporated into the equilibrium notion. This issue can be partly resolved by considering the implementability of the REE. The REE correspondence is not implementable due to problems that occur on the boundary of the feasible set identical to those encountered with the Walrasian correspondence. However, a suitably modified version of the REE, namely the Constrained Rational Expectations Equilibria (CREE), is indeed implementable under certain conditions.

This is closely related to Palfrey and Srivastava [8] where the implementability of SCC's that are of particular interest to economists, including the one induced by REE, was considered. The approach they employed was either to show a given SCC satisfies a set of sufficient conditions, as in the general implementability theorems, or to show that it violates a monotonicity condition which was shown to be necessary for implementation. A set of sufficient conditions for the implementability of REE was provided, and it was demonstrated that relaxing part of the conditions leads to non-implementabiltity. Example 4 in their paper stems from the issues raised by corner solutions and is not valid if one's attention is restricted to CREE. 
The mechanisms constructed in the general implementability theorems are quite complex and require the designer to know the individual preferences. Furthermore they are highly discontinuous and the informational structure in all the above theorems is assumed to be discrete with a finite number of possible states of the world.

The importance of the continuity property is stressed in Postlewaite and Wettstein [11] and Wettstein [13]. Discontinuous mechanisms suffer from the fault that a slight change in one's strategy which might be interpreted as a slight mistake, is liable to cause a considerable change in the outcome reached. A continuous mechanism also has the advantage that conclusions and suggestions are robust with respect to slight misspecifications.

In Wettstein [13] the implementability theorems were generalized to cover the case of a continuous informational structure, where the state of the world is given by the realization of a random variable which may, but need not, be discrete. The proofs were carried out by constructing an almost continuous mechanism. As a corollary one could get that the CREE, under certain conditions, are implementable in this more general setting as well. However the mechanism constructed still requires the designer to know the individual preferences.

It is thus natural to ask whether a particular scc can be implemented via a simpler mechanism, possessing nicer properties. Hurwicz [4], Schmeidler [12] and Postlewaite and Wettstein [11] answered this question when constructing mechanisms implementing the Walrasian (and variants thereof) correspondence in economies with complete information. We address this question in economies with incomplete information. 
In this paper we construct a feasible and almost continuous mechanism which, under certain conditions implements the CREE. It improves upon the previously suggested mechanisms in that it does not require the designer to know the individual preferences. The strategy space itself will be reminiscent of a market in which prices and quantities are announced. The feasibility requires the mechanism to provide a feasible outcome for any configuration of actions chosen by the participating individuals. We note that if one allows for nonfeasible outcomes outside of equilibrium, the REE in example 5 of Palfrey and Srivastava [8] can be implemented by an almost identical mechanism to the one we shall present below.

The mechanism constructed will use a signalling stage which in our opinion is a natural device in the modelling of market behavior under conditions of incomplete information and greatly simplifies the presentation. We shall make extensive use of the Non Exclusive Information (NEI) ${ }^{1}$ assumption which appeared in Postlewaite and Scmeidler [9] and [10]. In related work, Blume and Easley [2] have shown that violation of the NEI assumption leads to non implementability of the REE.

The second section describes the environments we consider and introduces notation and definitions. In the third section we construct a feasible and almost continuous mechanism implementing the CREE. The fourth and final section discusses possible extensions and further lines of research.

\section{Notation and Definitions}

$\left(R^{n}, B^{n}, \mu\right)$ - The $n$-dimensional Euclidean space viewed as a probability space with $B^{n}$ denoting the set of Borel sets and $\mu$ a probability measure defined on $B^{n}$. A generic element of this space will be denoted by: 


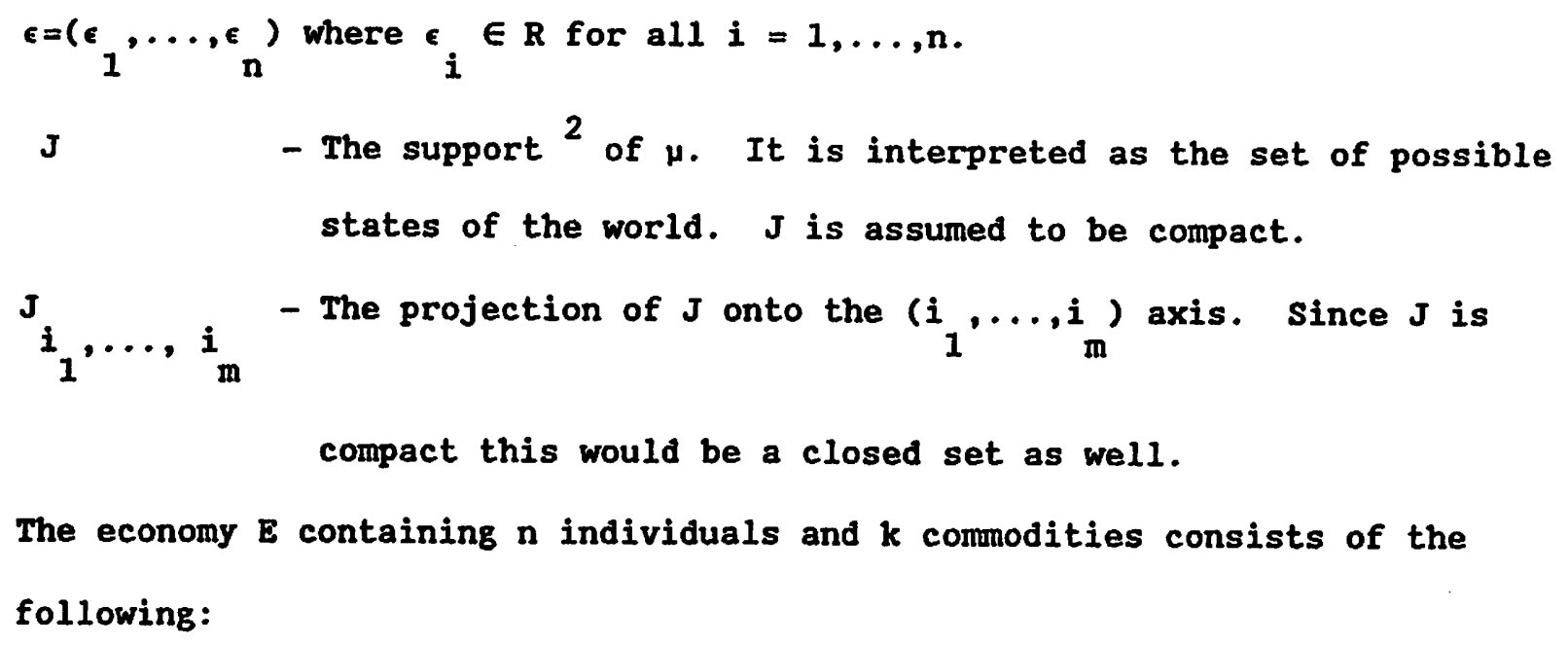

1. An n-tuple of von Neumann-Horgenstern utility functions $\mathrm{U}_{\mathrm{i}}: \mathrm{R}_{+}^{\mathrm{K}} \times \mathrm{J} \rightarrow \mathrm{R}$

$u_{i}(x, \epsilon)$ denotes the satisfaction individual $i$ derives from consuming bundle $x$, when the state of the world is given by $\epsilon$. The $U_{i}$ 's are assumed to be measurable ${ }^{3}$ in their last $n$ coordinates.

2. An $n$-tuple of initial endowments $\left(w_{1}, \ldots, w_{n}\right)$ where $w_{i} \in R_{+}^{K}$ for all $i=1, \ldots, n . \quad w=\sum_{i=1}^{n} w_{i}$.

Hence initial endowments are fixed and do not vary with the state of the world. If one were willing to consider nonfeasible implementation there would be no need to fix the initial endowments. This issue was treated extensively in Hurwicz, Maskin and Postlewaite [5].

We assume that the economy and the probability space are common knowledge as in Aumann [1], but individual $i$ observes $\epsilon_{i}$; this observation is private and gives rise to the asymetric information aspect.

A - The set of feasible allocations for $E$.

$A=\left\{\left(x_{1}, \ldots, x_{n}\right) \in R_{+}^{n K} \mid \sum_{i=1}^{n} x_{i}^{j} \leq \sum_{i=1}^{n} w_{i}^{j}\right.$ for $\left.j=1, \ldots, k\right\}$

f - An allocation rule defined as a measurable function associating a feasible allocation with each possible $\epsilon$. 
$f=\left(f_{1}, \ldots, f_{n}\right)$ where $f_{i}$ denotes the bundle allocated to individual $i$.

F - A Social Choice Correspondence (SCC) defined as a collection of allocation rules.

Under conditions of incomplete information, one should not preclude the possibility that the prices at which trade takes place partially (or completely) reveal the state of the world. In that case one would expect the individuals to use that additional information. This notion is formally captured in the following definition of a Rational Expectations Equilibrium.

A pricing function $P: J \rightarrow \mathbb{R}_{+}^{K}$ and an allocation rule $f$ constitute $a$ Rational Expectations Equilibrium (REE) if the following holds:

(i) $P$ is measurable.

(ii) $f_{i}$ is measurable with respect to $\epsilon_{i}$ and $P(\epsilon)^{4}$, for all $\mathbf{i}=1, \ldots, \mathrm{n}$.

(iii) For all $i=1, \ldots, n$ and almost everywhere (a.e.) in $J$ $f_{i}(\epsilon)$ solves: $\max _{i} E\left(U_{i}\left(x_{i}, \epsilon\right) / \epsilon_{i}, P(\epsilon)\right)$ S.T.

$$
\begin{aligned}
P(\epsilon) \cdot x_{i} & \leq P(\epsilon) \cdot w_{i} \\
x_{i} & \geq 0
\end{aligned}
$$

(iv) $P(\epsilon) \cdot\left(W-\sum_{i=1}^{n} f_{i}(\epsilon)\right)=0$ a.e. in J.

The conditional expectations are defined through the use of a conditional probability measure on $J$ given that the $i \frac{\text { th }}{}$ coordinate equals $\epsilon_{i}$ and $P$ equals $P(\epsilon)$. It is of course assumed the individuals know the probability space and the pricing function.

Due to problems created by corner solutions the REE correspondence is not implementable. This is taken care of by limiting our treatment to a 
Constrained Rational Expectation Equilibrium (CREE) which is defined as follows:

$P: J \rightarrow R_{+}^{K}$ and $f: J \rightarrow A$ constitute a CREE if the following holds:

(i). (ii) and (iv) as in the definition of an REE; (iii) is replaced by (iii)':

(iii)' for all $i=1, \ldots, n$ and a.e. in $J$,

$f_{i}(\epsilon)$ solves:

$\max _{i} E\left(U_{i}\left(x_{i}, \epsilon\right) \mid \epsilon, P(\epsilon)\right)$

S.T.

$$
\begin{aligned}
P(\epsilon) \cdot x_{i} & \leqq P(\epsilon) \cdot w_{i} \\
x_{i} & \leqq w \\
x_{i} & \leq 0
\end{aligned}
$$

Thus the difference between CREE and REE stems from the fact that in the former individuals are not allowed to demand a commodity bundle which exceeds in one or more of its coordinates the aggregate endowment vector. It is obvious that both defintions coincide for interior solutions.

Before outlining the specific mechanism constructed in this paper, we formally define the notions of a mechanism, Bayesian Nash Equilibrium (BNE) and implementation.

A mechanism $G$ will consist of the following:

1. An n-tuple of measurable strategy sets $\left(s_{1}, \ldots, s_{n}\right)$ $S_{i}=B_{i} \times H_{i} \times D_{i}$

$B_{i}$ denotes acts that have to be taken before the observation of any private information, $H_{i}$ denotes acts that are taken after the 
observation of the private information, but prior to observing any signals, while $D_{i}$ denotes acts taken after observing a signal sent by the designer.

$S=\prod_{i=1}^{n} s_{i} ; \quad B=\prod_{i=1}^{n} B_{i} ; \quad H=\prod_{i=1}^{n} H_{i} ; \quad D=\prod_{i=1}^{n} D_{i}$

2. A measurable signalling structure $P=\left(P_{1}, \ldots, P_{n}\right)$ where $P_{i}: B \times H \rightarrow \Gamma_{i}$

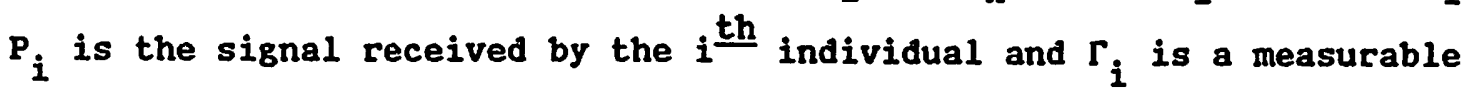
signal space

$\Gamma=\prod_{i=1}^{n} \Gamma_{i}$

3. A measurable outcome function

$$
\begin{aligned}
& B: B \times H \times D \rightarrow A \\
& g=\left(g_{1}, \ldots, g_{n}\right) \text { where } g_{i} \text { denotes the bundle received by } \\
& \text { individual } i .
\end{aligned}
$$

A strategy for individual $i$ would be a choice of $b_{i}$ in $B_{i}$, a measurable function $h_{i}: J_{i} \rightarrow H_{i}$ and a measurable function $d_{i}: J_{i} \times r_{i} \rightarrow D_{i}$.

In defining the BNE of such a mechanism we use the following notation:

$$
\begin{aligned}
& s=\left(s_{1}, \ldots, s_{n}\right) \\
& \quad \text { for } s \text { in } s_{i} \text { define }\left(s_{-i}, \hat{s}\right)=\left(s_{1}, \ldots, s_{i-1}, s_{i+1}, \ldots, s_{n}\right)
\end{aligned}
$$

A BNE of the mechanism is an n-tuple of strategies

$$
\begin{aligned}
& \tilde{s}=\left(\tilde{s}_{1}, \ldots, \tilde{s}_{n}\right) \text { where } \tilde{s}_{i}=\left(\tilde{b}_{i}, \tilde{h}_{i}, \tilde{d}_{i}\right) \text { satisfies for all } i=1, \ldots, n \\
& E\left(U_{i}\left(g_{i}(\tilde{s}), \epsilon\right)\right) \geq E\left(U_{i}\left(g_{i}\left(\tilde{s}_{-i}, \tilde{s}\right), \epsilon\right)\right) \text { for all } s \text { in } s_{i} .
\end{aligned}
$$

Note that this would imply that the strategy choice made for any $\epsilon_{i}$ and any signal subsequently observed is optimal given the choices made by others except, perhaps, on a set of $\epsilon_{i}$ 's and signals which has a probability zero of arising. 
The strategies chosen by the individuals yield an allocation rule a. $a(\epsilon)=g(s)$

$N_{G}(E)$ - The set of allocation rules corresponding to BNE of the mechanism for $\mathrm{B}$.

G implements $F$ if:

$$
N_{G}(B)=F \cdot a \cdot e^{5}
$$

The use of an explicit signalling stage is not essential; that type of a mechanism can be represented by a more complicated one which will involve no signalling. This can be done in the same way in which one moves from a game in extensive form to a game in strategic form. However the use of a signalling stage is only natural in the context of REE.

In the general implementability theorems the mechanism constructed indeed depends on the $U_{i}$ 's. The mechanism we shall construct below will work for an entire class of economies provided the $U_{i}^{\prime}$ s satisfy some technical conditions.

As remarked earlier, to achieve implementability we must assume the support of $\mu$ satisfies a Non Exclusive Information (NEI) assumption. It can be formulated in various ways and we formulate it as follows:

(NEI) $\sum_{i=1}^{n} c_{i}=0 \quad$ for all $\in$ in $J$.

Admittedly this is not the most general formulation possible, however all that is subsequently used is the fact any $(n-1)$ coordinates of 6 uniquely determine the remaining one.

3. The mechanism

The strategy space for individual i will be:

$$
s_{i}=B_{i} \times H_{i} \times D_{i}
$$




$$
\begin{aligned}
& \text { where }{ }_{i}=\tilde{P} \\
& r_{i}^{D_{i}}=\tilde{J}_{i} \\
& \tilde{P} \text { is the set of all measurable functions from } J \text { into } R_{+}^{K} \cdot \text { A generic }
\end{aligned}
$$

element of the strategy space will be denoted by $\left(P^{i}, r_{i}, z_{i}, m_{i}\right)$.

The first component belongs to $B_{i}$, and has to be decided upon before the observation of any private information.

The second component belongs to $\mathrm{H}_{i}$ and has to be decided upon after the observation of $\epsilon_{i}$. The last two components belong to $D_{i}$ and are decided upon after observing a signal which in our case will be some price vector.

The strategy of individual $i$ can be given the following interpretation: $p^{i}$ - The pricing rule individual $i$ would like to prevail.

$r_{i}$ - The $\epsilon_{i}$ he "observed".

$z_{i}$ - A net trade he would like to have.

$m_{i}$ - A number playing a dual role of determining how much weight should be assigned to the net trade demanded and how the fines imposed on individual $i$ (for any detected lies, $\epsilon$ 's outside $J$ ) are determined.

The outcome function is defined as follows:

\section{Stage 1}

Construct a weighted average of the $P^{i}$ 's and denote it by $\bar{P}$. $\bar{P}(\epsilon)$, the price vector corresponding to an $\epsilon$ in $J$, is constructed as follows: 
Define:

$$
a_{j}(\epsilon)=\sum_{t, t^{\prime} \neq j}\left|p^{t}(\epsilon)-p^{t^{\prime}}(\epsilon)\right|
$$

where $p^{t}$ denotes the pricing function announced by individual $t$. Now define: $\alpha=\sum_{j=1}^{n} \alpha_{j}$

and finally:

$$
\beta_{j}=\left\{\begin{array}{ll}
\alpha_{j} & \text { if } \alpha>0 \\
\frac{1}{n} & \text { if } \alpha=0
\end{array} \quad j=1, \ldots, n\right.
$$

$$
\bar{P}(\epsilon)=\sum_{j=1}^{n} \beta_{j} P^{j}(\epsilon)^{6}
$$

From the construction of $\bar{P}$ it is clear that if all the individuals announce the same pricing function then no single individual can change the $\bar{P}$ arrived at by changing his own announcement.

\section{Stage 2}

This stage will involve several projections and care must be taken to ensure all these operations are measurable. There are $n$ closed sets (in $R^{n-1}$ ) $J_{1,2, \ldots, i-1, i+1, \ldots, n}$ for $i=1, \ldots, n$ on which we need to project tuples of numbers announced by the individuals. Prior to starting the operation of the mechanism, n measurable functions (one for each set) carrying out these projections are constructed. When the sets in question are convex the projection operation yields a continuous and thus certainly measurable function. If the sets are not convex the projection operation turns out to be a correspondence. The problem is resolved by lemma 1 in Hildenbrand 
[3, p. 55] which shows it is possible to select a measurable function out of such a correspondence. These $\mathrm{n}$ functions are assumed to be common knowledge among the individuals.

Those functions are used by the mechanism to construct $n$ profiles in $J$. The $i^{\underline{t h}}$ profile denoted by $\left(\tilde{r}_{1}^{i}, \ldots, \tilde{r}_{n}^{i}\right)$ is constructed in the following manner.

The projection (by the above mentioned function) of $\left(r_{1}, \ldots, r_{i-1}, r_{i+1}, \ldots, r_{n}\right)$ on $J_{1}, \ldots, r_{i-1, i+1}, \ldots, n$ is denoted by $\left(\tilde{r}_{1}^{i}, \ldots, \tilde{r}_{i-1}^{i}, \tilde{r}_{i+1}^{i}, \ldots, \tilde{r}_{n}^{i}\right)$ and $\tilde{r}_{i}^{i}$ is defined by $\tilde{r}_{i}^{i}=-\sum_{j \neq i} \tilde{r}_{j}^{i}$

This way we indeed get a profile in $J$ given by:

$$
\tilde{r}^{i}=\left(\tilde{r}_{1}, \ldots, \tilde{r}_{\mathbf{i}}\right)
$$

The following two facts are evident from the construction

(i) If $\left(r_{1}, \ldots, r_{n}\right)$ is in $J$ then all the $n$-profiles are identical and equal to $r$.

(ii) A change in the strategy of individual $i$ will not change the $i$ th profile.

At this point we have a pricing function $\bar{p}$ and $n$ profiles $\left(\tilde{r}^{i}\right)_{i=1}^{n}$.

Individual $i$ is now told what $P\left(\tilde{r}^{i}\right)$ is, $i . e$. he is told a certain price vector in $R_{+}^{K}$. 


\section{Stage 3}

Having been told their respective price vectors, the individuals send their $z_{i}^{\prime}$ s and $m_{i}$ 's. A set $k^{i}$ is defined for each individual.

$$
k^{i}\left(\bar{P}\left(\tilde{r}^{i}\right), w_{i}, w\right)=\left\{\begin{array}{l|l}
z \in R^{K} & \begin{array}{l}
P\left(\tilde{r}^{i}\right) \cdot z=0, z+w_{i} \geq 0 \\
z+w_{i} \leq w
\end{array}
\end{array}\right\}
$$

$y_{i}$ is defined to be the closest point in $k^{i}$ to $z_{i}$. Once again a projection is made; but since $x^{i}$ is a closed and convex set this is continuous and well defined.

$$
\begin{aligned}
& \text { Next the mechanism defines } \\
& c=\left\{\begin{array}{l|l}
m \in R_{+} & \begin{array}{l}
m \cdot m_{i} \leq 1 \\
\text { and } \\
m \cdot \sum_{i=1}^{n} m_{i}\left(y_{i}+w_{i}\right) \leq w
\end{array}
\end{array}\right\}
\end{aligned}
$$

$\mathrm{m}^{\star}$ will be defined by $\mathrm{m}^{*}=\max _{\mathrm{m} \in \mathrm{C}} \mathrm{m}$

For the last step we need to perform one more projection, this time on J. Similar to our previous discussion we use a measurable projection function constructed prior to the game and assumed to be common knowledge among the individuals.

Project $\left(r_{1}, \ldots, r_{n}\right)$ on $J$ and get a point $\epsilon^{\star}$ in $J$ (closest to

$$
\begin{aligned}
& \left.\left(r_{1}, \ldots, r_{n}\right)\right) \text { and let: } \\
& \quad g_{i}\left(s_{1}, \ldots, s_{n}\right)=m^{*} \cdot m_{i} t_{i}\left(y_{i}+w_{i}\right)
\end{aligned}
$$

where:

$$
t_{i}=\left(1+\frac{\sum_{j=1}^{n} m_{j}}{m_{i}}\left|r-\epsilon^{*}\right|\right)^{-1}
$$


Theorem: If the following assumptions are satisfied, then the mechanism described above implements the CREE.

(AI) $n \geq 3$

(A2) For all $i=1, \ldots, n U_{i}$ is continuous, strictly increasing and strictly concave in its first $K$ arguments for any $c$ in $J$.

(A3) For all $i=1, \ldots, n \quad U_{i}(W, \epsilon)$ is integrable in its last $n$ arguments.

(A4) For all $i=1, \ldots, n \quad w_{i}$ is in $R_{++}^{K}$

(A5) J satisfies NEI.

Proof: In the first part of the proof we show that any CREB allocation rule can arise up to a set of measure zero as a BNE of this mechanism.

Let $P(\epsilon)$ and $f(\epsilon)$ denote a CREE.

We now construct the following BNE which yields $f$.

$p^{i}=p$ for all $i=1, \ldots, n$

$r_{i}=\epsilon_{i}$ for all $\epsilon$ in $J$ and all $i=1, \ldots, n$

$z_{i}=f_{i}(\epsilon)-W_{i}$ for all $\epsilon$ in $J$, all possible signals arising out of

$m_{i}=1 \quad$ the $P$ and $r$ choice, and all $i=1, \ldots, n$.

In the first stage we obtain $P$ and no single individual can change it by deviating and declaring some other pricing function.

The second stage yields the true profile $\left(\epsilon_{1}, \ldots, \epsilon_{n}\right)$ for all the individuals, and each individual is told the true price $P\left(e_{1}, \ldots, \epsilon_{n}\right)$.

For almost every $c$ in $J, z_{i}$ belongs to $k^{i}$ and hence $y_{i}=z_{i}$ a.e. in $J$.

since $f$ was a CREE we have that for a.e. 6 in $J$.

$\sum_{i=1}^{n} f_{i}(\epsilon)=W$

and hence for a.e. $c$ in $J$ 


$$
\sum_{i=1}^{n}\left(y_{i}+w_{i}\right)=w
$$

since $m_{i}=1$ for all $i=1, \ldots, n$ we get $m^{*}=1$ for a.e. 6 in $J$.

Since individuals report their true $\epsilon_{i}, t_{i}=1$ for all $\epsilon$ in $J$. Thus

$8\left(s_{1}, \ldots, s_{n}\right)=f(\epsilon)$ a.e. in J.

Now we must show that this n-tuple of strategies does form a BNE.

Individual $i$ cannot change the pricing function, the signal he gets or the set $k^{i}$

By the definition of a CREE his strategy choice gives him for almost every $\epsilon_{i}$ and signal observed the most preferred point in the set $k^{i}$. This is true for all individuals $i=1, \ldots, n$ and hence this $n$-tuple of strategies forms a BNE.

Now we shall show that any BNE of the game coincides, up to a set of measure zero, with some CREE allocation rule.

A BNE gives rise to some pricing function $P$ which is constructed in stage 1. At the second stage we have an n-tuple of measurable strategies. $r_{1}\left(\epsilon_{1}\right), \ldots, r_{n}\left(\epsilon_{n}\right)$ where $r_{i}: J_{i} \rightarrow J_{i}$

These functions show what observation individual $i$ would report as a function of his true observation. Because of the " $t_{i}$ term" we get that the mapping

$$
r(\epsilon)=\left(r_{1}\left(\epsilon_{1}\right), \ldots, r_{n}\left(\epsilon_{n}\right)\right)
$$

must satisfy $r(\epsilon)$ is in $J$ for a.e. $\epsilon$ in $J$, otherwise no choice of $m_{i}$ could serve as an equilibrium strategy. Individuals would like to announce infinitely large $m_{i}$ 's.

We denote by $J$ the set of $\epsilon^{\prime} s$ for which $r(\epsilon)$ is in $J$, and restrict our attention from now on to $\mathrm{J}$. 
The "effective" pricing function on $\hat{J}$ is

$$
\bar{P}(\epsilon)=P(r(\epsilon))
$$

After stage 2 individual i's expected utility of consuming comodity bundle $x_{i}$ is denoted by $v_{i}\left(x_{i}\right)$ where:

$$
v_{i}\left(x_{i}\right)=E\left(\left.U_{i}\left(x_{i}, \epsilon\right)\right|_{i}, \bar{P}(\epsilon)\right)
$$

Now we show that for a.e. $\epsilon$ in $J$, for all $i=1, \ldots, n$,

$$
\begin{aligned}
& m^{*} \cdot m_{i}=1 \\
& \text { and } \\
& \sum_{i=1}^{n}\left(y_{i}+w_{i}\right)=\sum_{i=1}^{n} w_{i}
\end{aligned}
$$

This replicates arguments which appeared in Postlewaite and Wettstein [11] and is given here in detail to make this presentation self contained. Suppose by way of contradiction there exist an individual $i$ and a set $D$ of positive measure in $J$ for which $m^{*} \cdot m_{i}<1$. In this case individual $i$ could improve his position by announcing a larger $m_{i}$ for all $\epsilon_{i}$ in the projection of $D$ on the $i^{t h}$ axis. (Precise calculations justifying this claim are presented in Postlewaite and Wettstein [11]). This contradicts the fact $\mathrm{m}_{i}$ formed part of a BNE, since there exists a set of positive measure over which the individual could have done better.

So we have shown $\mathrm{m}^{\star} \cdot \mathrm{m}_{i}=1$ a.e. in $\hat{J}$, this fact will now be utilized in proving the second claim. 
Suppose by way of contradiction there exists a set of positive measure

in $\mathrm{J}$ for which

$$
\sum_{i=1}^{n}\left(y_{i}+w_{i}\right) \neq \sum_{i=1}^{n} w_{i}
$$

By the definition of $C, \sum_{i=1}^{n}\left(y_{i}+w_{i}\right) \leq \sum_{i=1}^{n} w_{i}$ for a.e. $\epsilon$; hence there

must be a coordinate $s$ and a set $D$ of positive measure, in $\vec{J}$ for which

$$
\sum_{i=1}^{n} y_{i}^{s}+w_{i}^{s}<\sum_{i=1}^{n} w_{i}^{s}
$$

However since $y_{i}$ is for all $i=1, \ldots$ n for a.e. $\epsilon$ in $x^{i}$, we have

$$
P\left(\sum_{i=1}^{n} y_{i}+w_{i}\right)=P \sum_{i=1}^{n} w_{i} \text { for a.e.e in } J .
$$

Hence there must be a coordinate $s^{\prime}$ and a set of positive measure in J for which:

$$
\sum_{i=1}^{n}\left(y_{i}^{s^{\prime}}+w_{i}^{s^{\prime}}\right)>\sum_{i=1}^{n} w_{i}^{s^{\prime}}
$$

which once more contradicts the definition of $c$.

Thus we have for a.e. $\epsilon$ in $J$

$$
\sum_{i=1}^{n}\left(y_{i}+w_{i}\right)=\sum_{i=1}^{n} w_{i}
$$

Continuing as in Postlewaite and Wettstein [11] we show that for any 6 in $\bar{J}$ the $i^{\frac{t h}{}}$ individual can get arbitrarily close to any net trade $z$ that satisfies:

$$
\text { (i) } \bar{P}(\epsilon) \cdot z=0
$$




$$
\begin{aligned}
& \text { (ii) } z+w_{i} \leq w \\
& \text { (iii) } z+w_{i} \geq 0
\end{aligned}
$$

This can be done on observing $\bar{P}(\epsilon)$, by announcing a large enough $m_{i}$ that will make $\mathrm{m}^{\star}$ arbitrarily small. This will nullify the effect of other terms in the sum $\mathrm{m}^{*} \cdot \sum_{i=1}^{n} m_{i}\left(y_{i}+w_{i}\right)$. Thus on observing $\bar{P}(c)$, announcing $z$ which yields $y_{i}$ equal to $z$ will bring individual $i$ arbitrarily close to the $z$ net trade.

Finally, we need to prove that $\bar{P}(\epsilon)$ and the allocation rule yielded by the mechanism at the BNE point, denoted by $f(\epsilon)$, form part of a CREE. Since in the definition of a CREE everything holds up to a set of measure zero, we are justified in restricting our attention to $\mathrm{J}$.

$$
\begin{aligned}
& \text { We shall show that a.e. in } \bar{J} \quad f_{i}(\epsilon) \text { solves the problem: } \\
& \max _{i} v_{i}\left(x_{i}\right) \\
& \text { S.T. }
\end{aligned}
$$

$$
\begin{gathered}
\bar{P}(\epsilon) x_{i} \leq \bar{P}(\epsilon) w_{i} \\
x_{i} \leq w \\
x_{i} \geq 0
\end{gathered}
$$


By the definition of $k^{i}$ and the fact $m^{*} \cdot m_{i}=1$ for a.e. $\epsilon$ in $J, f_{i}(\epsilon)$ is feasible for the problem for a.e. $\epsilon$ in $\mathrm{J}$.

Assume by way of contradiction there exists a set $D$ of positive measure in $J$, such that an allocation rule which is feasible for problem

(1) and measurable with respect to $\epsilon_{i}$ and $\bar{P}(\epsilon)$ yields a bundle individual i strictly prefers to $f_{i}(\epsilon)$ for all $\epsilon$ in $D$. By strict monotonicity of preferences we may assume, without loss of generality, that all those bundles lie on the budget line. Then as we have shown the $i^{\text {th }}$ individual can, by a suitable change of strategies, for the appropriate $\epsilon_{i}$ 's and $\overline{\mathbf{P}}(\epsilon)$ 's get arbitrarily close to those bundles. By assumption (A3) we can use the Lebesque Dominated Convergence Theorem and show that since the $U_{i}$ 's are continuous (assumption $\left(A_{2}\right)$ ), the $v_{i}$ 's are continuous as well. But the continuity of $v_{i}$ implies the individual could make himself better off on a set of positive measure, contradicting the fact we started from a BNE.

Thus for all $i=1, \ldots, n$ and a.e. in $J f_{i}(\epsilon)$ solves problem (1).

In conclusion we have shown that the allocation rule yielded by the mechanism coincides a.e. in $J$ (and hence in $J$ ) with a CREE allocation rule.

\section{Concluding Remarks}

We have successfully implemented, constructing an almost continuous mechanism, the SCC induced by CREE. The mechanism constructed, in contrast 
with previous mechanisms does not require the designer to know the $U_{i}$ 's and performs well for a large class of economies. The mechanism will be truly continuous if all the projections performed can be carried out in a continuous fashion. This will be the case if $J$ is a convex set.

One undesirable feature of this mechanism is the nonexistence of a best response strategy for some parts of the strategy space. This could be avoided by using well known punishment schemes if a lie is detected but then we lose the continuity feature which we deem to be quite important. The NEI assumption is admittedly very restrictive; however it seems to be unavoidable in this framework.

We have used a deterministic signalling stage, one could also conceive of random signalling schemes to capture the idea that individuals observe noisy information.

Incorporating production into the pure exchange framework is a natural extension and the subject for future research. The presence of incomplete information which in a sense is similar to the phenomena of incomplete markets creates several difficult issues which do not arise in the usual walrasian framework. 


\section{Footnotes}

1. The NEI assumption states that the pooled information of any set of $n-1$ individuals, where $\mathrm{n}$ is the total number of individuals, is complete.

2. The support of $\mu$ is the smallest closed set in $R^{n}$ with measure 1 .

3. Unless otherwise specified, measurability is taken to be in the Borel sense.

4. Thus the values $f_{i}$ assumes for any $\epsilon$ in $J$ can depend only on the $i$ th coordinate of $\epsilon$ and the associated price vector.

5. Any allocation rule in $N_{G}(E)$ is equal to some allocation rule in $F$ almost everywhere, and vice versa.

6. We note that $\bar{P}(\epsilon)$ is a continuous function of the $P^{j}$ 's even though the $\beta_{j}$ 's are not. 


\section{References}

1. R.J. Aumann, Agreeing to disagree, Ann. Statist. 4 (1976), 1236-39.

2. L. Blume and D. Easley, Implementation of expectations equilibria, Univ. of Michigan mimeo, 1985.

3. W. Hildenbrand, "Core and Equilibria of a Large Economy," Priceton Univ. Press, Princeton, NJ. 1974.

4. L. Hurwicz, Outcome functions yielding Walrasian and Lindahl allocations at Nash Equilibrium points, Rev. Econ. Stud. 46 (1979), 217-25.

5. L. Hurwicz, E. Maskin and A. Postlewaite, Feasible implementation of social choice correspondences by Nash equilibria, mimeo, 1982.

6. J. Jordan and R. Radner, Rational Expectations in microeconomic models: An overview, J. Econ. Theory 26 (1982), 201-23.

7. T. Palfrey and S. Srivastava, Implementation with incomplete information, Carnegie Mellon Univ. mimeo, 1985.

8. T. Palfrey and S. Srivastava, On Bayesian implementable allocations, Rev. Econ. Stud. 54 (1987), 193-208.

9. A. Postlewaite and D. Schmeidler, Revelation and implementation under differential information, Univ. of Pennsylvania mimeo, 1983.

10. A. Postlewaite and D. Schmeidler, Implementation in differential information economies, J. Econ. Theory 39 (1986), 14-33.

11. A. Postlewaite and D. Wettstein, Feasible and continuous implementation of constrained Walrasian equilibria, Univ. of Pennsylvania mimeo, 1982.

12. D. Schmeidler, Walrasian analysis via strategic outcome functions, Econometrica $48(1980), 1585-93$.

13. D. Wettstein, "Implementation Theory in Economies with Incomplete Information," Doctoral Dissertation, Univ. of Minnesota, Minneapolis, 1987. 